\title{
Non-integrability of a system with the Dyson Potential
}

\author{
Georgi Georgiev
}

\begin{abstract}
In this paper it is shown that the Hamiltonian system with Dyson potential is analytical non-integrable and formal non-integrable. The approach is based on the following: when a system has a family of periodic solutions around an equilibrium and if the period function is infinitely branched then the system has non additional analytic first integral. We prove formal non-integrability using Ziglin-Moralez-Ruiz-Ramis theory.
\end{abstract}

Keywords: Dyson potential, Hamiltonian systems

2010 MSC: 70H05, 70H07, 34M55, 37J30

We study the Hamiltonian system of $n$ interacting particles of equal mass with a Hamiltonian

$$
H=\frac{1}{2} \sum_{i=1}^{n} y_{i}^{2}+\sum_{i<j} V\left(x_{i}-x_{j}\right), V(x)=-\log |\sin x| .
$$

Here $x_{1}, x_{2}, \ldots x_{n}$ are the coordinates of particles, $y_{1}, y_{2}, \ldots y_{n}$ are their momenta, and $V$ is the potential energy in Dyson type. The system with this potential was studied in [1], where the statistical properties of the energy levels of one dimensional Coulomb's gas are investigated.There is a connection between system of point vortices and regarding system in [2] by Calogero and Perelomov. The equilibria position of the system (0.1), $x_{k}^{0}=x_{0}+\frac{\pi k}{n}, k=$ $1,2, \ldots n, x_{0} \in \mathbf{R}$ determines stationary collinear choreography on the sphere-point vortices are located in the equatorial plane that uniformly rotates around an axis lying in this plane.

The function $V$ is $2 \pi$-periodic (and even $\pi$-periodic), so we can assume that the particles move in circles. The Hamiltonian system always has two integrals

$$
H, F=\sum_{i=1}^{n} y_{i}
$$

The question of integrability of (0.1) is studied by Calogero and Perelomov [2]. If $V$ is a non-constant analytic periodic function without singularities, then the system (0.1) can not be integrable for $n \geq 3$ ( Kozlov [3]). Unfortunately Dyson potential has a real logarithmic singularity. Borisov and Kozlov in [4] had proved that the system in case $n=3$ is nonintegrable in analytical first integrals. We consider the same case $n=3$, but the approach is 
different, this is the first non-trivial case and we prove that the system admits only $F$ as a holomorphic first integral in a complex domain.

Let us make in (0.1) (in our case $n=3$ ) canonical change of variables

$$
\begin{aligned}
& y_{1}=p_{1}+p_{3}, y_{2}=-p_{1}+p_{2}+p_{3}, y_{3}=-p_{2}+p_{3}, \\
& q_{1}=x_{1}-x_{2}, q_{2}=x_{2}-x_{3}, q_{3}=x_{1}+x_{2}+x_{3},
\end{aligned}
$$

and we obtain

$$
H=p_{1}^{2}-p_{1} p_{2}+p_{2}^{2}+\frac{3}{2} p_{3}^{2}-\log \left|\sin q_{1}\right|-\log \left|\sin q_{2}\right|-\log \left|\sin \left(q_{1}+q_{2}\right)\right| .
$$

The system has another integral

$$
F=3 p_{3}
$$

and has a stable equilibrium $p_{1}=p_{2}=0, q_{1}=q_{2}=\frac{\pi}{3}$.

The variable $q_{3}$ is cyclic and it reduces the system (with $p_{3}=$ const) to a system with 2-degrees of freedom with Hamiltonian

$$
H_{\text {reg }}=p_{1}^{2}-p_{1} p_{2}+p_{2}^{2}-\log \left|\sin q_{1}\right|-\log \left|\sin q_{2}\right|-\log \left|\sin \left(q_{1}+q_{2}\right)\right| .
$$

We need some basic definitions about Hamiltonian systems.

Let $H$ is a smooth real-valued function of $2 n$ real variables $(p, q), p, q \in \mathbf{R}^{\mathbf{n}}$. Let us also assume that $d H(0)=0$ where 0 is an equilibrium point for the Hamiltonian system $X_{H}$ (with $n$ degrees of freedom), given by

$$
\frac{d q}{d t}=\frac{\partial H}{\partial p}, \frac{d p}{d t}=-\frac{\partial H}{\partial q}
$$

We often write the Hamiltonian systems in the form

$$
\dot{x}=X_{H}(x), x \in \mathbf{R}^{2 \mathbf{n}},
$$

where $X_{H}$ is the flow. The system is called Liouville - Integrable near 0 if there exists $n$ functions in involution $f_{1}=H, f_{2}, \ldots f_{n}$, defined around 0 , are functionally independent. The Poisson bracket of $f$ and $g$ are

$$
\{f, g\}=X_{f}(g)=\sum \frac{\partial f}{\partial p} \frac{\partial g}{\partial q}-\frac{\partial f}{\partial q} \frac{\partial g}{\partial p}=-\{g, f\}
$$

We call that functions $f$ and $g$ are in involution if the Poisson bracket is commuteve. This means that $d f_{1}, d f_{2}, \ldots d f_{n}$ are linearly independent around the equilibrium 0 and $f_{j}=$ const for all $j$ define smooth submanifolds, these level manifolds are invariant under $X_{f_{i}}$. We have $X_{f_{j}} f_{j}=0$ and $\left[X_{f_{j}}, X_{f_{k}}\right]=X_{\left\{f_{j}, f_{k}\right\}}=0$ - these flows commute. The compact and connected component of $M_{c}:=\left\{f_{j}=c_{j}, j=1, \ldots, n\right\}$ is diffeomorphed to a torus. 
We call that the system with Hamiltonian $H$ formally integrable if there exist formal power series $\tilde{f}_{1}, \tilde{f}_{2}, \ldots \tilde{f}_{n}$ in involution, where $\tilde{f}_{1}, \tilde{f}_{2}, \ldots \tilde{f}_{n}$ are functionally independent and Taylor expansion $\tilde{H}$ of $H$ is a formal power series in $\tilde{f}_{1}, \tilde{f}_{2}, \ldots \tilde{f}_{n}$. An asymptotic behavior near equilibrium is like an integrable system. The formal integrability gives information about the flow. The functional independence of $\tilde{f}_{1}, \tilde{f}_{2}, \ldots \tilde{f}_{n}$ is stronger than the independence of smooth functions $f_{1}, f_{2}, \ldots f_{n}$ of which $\tilde{f}_{1}, \tilde{f}_{2}, \ldots \tilde{f}_{n}$ are the Taylor series, because formal independence leads to a functional independence of a finite part of Taylor expansions.

Here we study the formal non-integrability of the system (0.1) in case $n=3$.

Our first aim is the following

Theorem 1. a) The system with Hamiltonian (0.4) is not integrable by means of analytical first integral;

b) The system with Hamiltonian(0.4) is not formal integrable.

The motivation for proving formal non-integrability I received from the remarcable paper of J. J. Duistermaat [5]. The Hamiltonian system with Dyson potential has similar structure. The proof of a) is different from [4].

Proof a): The proof of this is based on three propositions. The first - shows that there exists family of periodical solutions near equilibrium. The second proposition investigates the behavior on period function of these periodical solutions around the equilibrium. The third proves that if we assume an existence of an additional first integral- it is a constant.

Proposition 1. On the manifold $P:=\left\{p_{1}=p_{2}, q_{1}=q_{2}\right\}$, invariant under $X_{H}$ the system with Hamiltonian (0.4) exists a family of periodical solutions around equilibrium $p_{1}=p_{2}=0$, $q_{1}=q_{2}=\frac{\pi}{3}$.

Proof: Let we take $q(t):=q_{1}(t)=q_{2}(t), p(t):=p_{1}(t)=p_{2}(t)$ and $\frac{d q(t)}{d t}=p(t)$ in our system, then

$$
\dot{p}=\cot q+\cot 2 q,
$$

and we have

$$
(\dot{q})^{2}=\log (\sin 2 q)+2 \log (\sin q)+E .
$$

This is a conservative system with convex potential

$$
\tilde{V}(q)=-\log (\sin 2 q)-2 \log (\sin q),
$$

and we know that for these predictions the system has a periodical solution around the equilibrium for fixed energy $H_{r e g}=E$ (see Figure 1). 


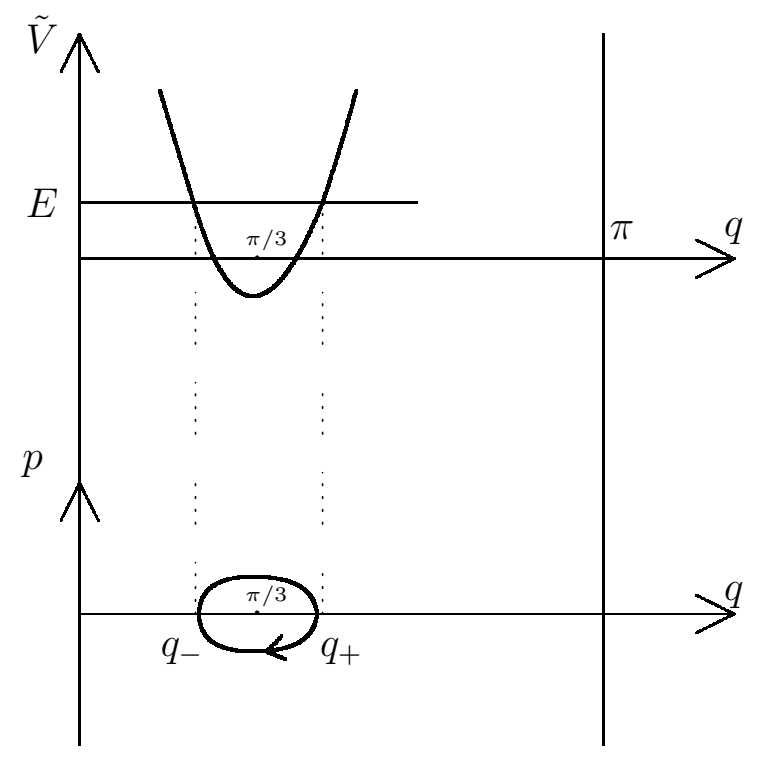

Figure 1: Periodical solution.

Proposition 2. The period function has expression $T(c)=\log \eta(c)+\Phi(c)$, where $c=\frac{1}{2 e^{E}}$, $\eta(c)=\epsilon(B(c)) \delta(B(c))$ with

$$
B(c)=\frac{16 \cdot\left(\frac{2}{3}\right)^{1 / 3} \cdot c^{2}}{\left(9 c^{2}-\sqrt{3} \sqrt{27 c^{4}-256 c^{6}}\right)^{1 / 3}}+2\left(\frac{2}{3}\right)^{1 / 3} \cdot\left(9 c^{2}-\sqrt{3} \sqrt{27 c^{4}-256 c^{6}}\right)^{1 / 3} .
$$

$\Phi(c)$ is an analytical function of the variable $c$ and

$$
r_{1,2}=\frac{1}{2}-\frac{1}{2} \sqrt{1+B} \pm \frac{1}{2} \sqrt{2-B+\frac{2}{\sqrt{1+B}}}
$$

$\epsilon=\frac{1}{2}\left(\arccos r_{1}-\frac{\pi}{3}\right), \delta=\frac{1}{2}\left(\frac{\pi}{3}-\arccos r_{2}\right)$.

Proof: The period in this solution is

$$
T=2 \int_{q_{-}}^{q_{+}} \frac{d q}{\sqrt{E-\tilde{V}(q)}}
$$

where $q_{-}$and $q_{+}$are the roots of $E-\tilde{V}(q)=0$, where $\tilde{V}(q)$ is (0.5). 
For equilibrium point $q=\frac{\pi}{3}$ we have $\tilde{V}\left(\frac{\pi}{3}\right)=-3 \log \left(\frac{\sqrt{3}}{2}\right)$ and let we fix $q_{+}=\frac{\pi}{3}+\epsilon$ and $q_{-}=\frac{\pi}{3}-\delta$, for $\epsilon>0$ and $\delta>0$. We have

$$
A=\frac{2}{\sqrt{E-\tilde{V}(q)}}=\frac{2}{\sqrt{-3 \log \left(\frac{\sqrt{3}}{2}\right)+\log (\sin 2 q)+2 \log (\sin q)}}
$$

The expansion of $A$ near $\frac{\pi}{3}$ is

$$
A=\frac{1}{\left|q-\frac{\pi}{3}\right|}-\frac{1}{3 \sqrt{3}}-\frac{2}{9}\left|q-\frac{\pi}{3}\right|+O\left(\left|q-\frac{\pi}{3}\right|^{2}\right) .
$$

For the period we obtain

$$
\begin{aligned}
T & =\int_{\frac{\pi}{3}-\delta}^{\frac{\pi}{3}+\epsilon} \frac{d q}{\sqrt{E-\tilde{V}(q)}}=\int_{\frac{\pi}{3}-\delta}^{\frac{\pi}{3}+\epsilon}\left(\frac{1}{\left|q-\frac{\pi}{3}\right|}-\frac{1}{3 \sqrt{3}}-\frac{2}{9}\left|q-\frac{\pi}{3}\right|+\ldots\right) d q \\
& =\log (\epsilon)+\log (\delta)+\Phi(\epsilon, \delta)=\log (\epsilon . \delta)+\Phi(\epsilon, \delta)=\log \eta+\Phi(\epsilon, \delta),
\end{aligned}
$$

where $\eta=\epsilon \delta$ and $\Phi(\epsilon, \delta)$ is an analytical function on each of the variables $\epsilon$ and $\delta$. Next we find $\epsilon$ and $\delta$. We have

$$
E=\tilde{V}(q)=-\log (\sin 2 q)-2 \log (\sin q)=-\log \left(2 \cdot(\sin q)^{3} \cdot \cos q\right),
$$

and if we get $q_{+}=\frac{\pi}{3}+\epsilon\left(\right.$ or $\left.q_{-}=\frac{\pi}{3}-\delta\right)$, then $-\left(\sin \left(\frac{\pi}{3}+\epsilon\right)\right)^{3} \cdot \cos \left(\frac{\pi}{3}+\epsilon\right)=\frac{1}{2 e^{E}}=c$. If we put $r=\cos \left(\frac{2 \pi}{3}+2 \epsilon\right)$, we obtain equation $(1-r)^{2}\left(1-r^{2}\right)=16 c^{2}$. The real roots of this equation are (0.7) with (0.6). We find $\epsilon=\frac{1}{2}\left(\arccos r_{1}-\frac{\pi}{3}\right), \delta=\frac{1}{2}\left(\frac{\pi}{3}-\arccos r_{2}\right)$.

Proposition 3. The system with Hamiltonian (0.4) does not possess any additional holomorphic first integral.

Proof: It is important for the proof that there is a family of solutions on $P$, of the Hamiltonian system (0.4) on the hypersurface $H=E$ are periodic. If $T$ is the period function then complex continuation of the manifolds $T=$ const turns out to be infinitely branched. This excludes the existence of a nontrivial analytic integral on any open subset of the complex domain where this infinite branching is true. Further, we need to show that if $G$ is a smooth function on open set $U$ such that $V=U \cap(H=E)$ is $X_{H}$ invariant, $\{H, G\}=0$ and derivative $d\{H, G\}=0$ on $V$, then $G$ is a function of $H$ and $T$ on $V$. The function $T(x), x \in \tilde{U} \subset U$ is real analytical and it is not constant $P$, it means that $d T \neq 0$ an open dense subset $\Delta$ of the manifold $P, G$ is invariant under flows $X_{H}$ and $X_{T}$. We have $X_{G} T=0$ on $V$. Further 
we regard as Hamiltonian flow on $P$ of any smooth extension $\tilde{T}$ on $T$ on open neighborhood of $P$, the flow is independent of the choice of the extension. On the open set $\Delta \cap V, d T$ and $d H$ are linearly independent that is why the flows $X_{H}$ and $X_{T}$ walk around one-dimensional submanifold $P, H=E$ and $T=$ const. Therefore on $\Delta \cap V, G$ is locally constant on $H=E$, and $T=$ const. That is $G$ function of $H$ and $T$ on the connected component of $\Delta \cap U \cap P$.

Let suppose that $G$ is analytic on $U$ and it has complex analytic extension on $\tilde{U}$ of $U$. If $G$ is not functionally depenended on $H$ on $P$ then the manifold $P$ and $G=$ const extend to closed complex analytic manifold on $\tilde{U}$, which coincides with complex analytic continuations of $H=E$ and $T=$ const. If the analytic continuations have infinite branching near $x \in \tilde{U}$, then we have a contradiction, so $G$ has to be a function of $H$ on $P$. Now we use $H$ as a coordinate near x, we can write $G=G_{0}+G_{1} H, G_{0}$ is a function of $H$ and $G_{1}$ is analytic. $G_{1}$ commutes with $H$ that is why $G_{1}$, this gives us that $G_{1}$ is a function of $H$ on $P$. We obtain that $G$ is a function of $H$ near $x$. By the analytic continuation this is true in the connected component of $x \in U$.

Proof b): Let us go back to the formal non-integrability. First we will regard the case $K=H_{2}+H_{3}$ it is Taylor expansion to a degree 3 with change of variables $\tilde{p_{1}}=p_{1}, \tilde{p_{2}}=p_{2}$, $\tilde{q_{1}}=q_{1}-\frac{\pi}{3}$ and $\tilde{q_{2}}=q_{2}-\frac{\pi}{3}$ (we move equilibrium to 0 )

$$
K={\tilde{p_{1}}}^{2}-\tilde{p_{1}}{\tilde{p_{2}}}+{\tilde{p_{2}}}^{2}+\frac{4}{3}{\tilde{q_{1}}}^{2}+\frac{4}{3} \tilde{q_{1}} \tilde{q_{2}}+\frac{4}{3}{\tilde{q_{2}}}^{2}+\frac{4}{9} \sqrt{3}{\tilde{q_{1}}}^{2}{\tilde{q_{2}}}+\frac{4}{9} \sqrt{3}{\tilde{q_{1}}}_{\tilde{q}_{2}}^{2}
$$

Let us remove tildes and we obtain the Hamiltonian system

$$
\begin{aligned}
& \dot{q_{1}}=2 p_{1}-p_{2} \\
& \dot{q_{2}}=-p_{1}+2 p_{2} \\
& \dot{p_{1}}=-\frac{8}{3} q_{1}-\frac{4}{3} q_{2}-\frac{8}{9} \sqrt{3} q_{1} q_{2}-\frac{4}{9} \sqrt{3} q_{2}{ }^{2} \\
& \dot{p_{2}}=-\frac{4}{3} q_{1}-\frac{8}{3} q_{2}-\frac{8}{9} \sqrt{3} q_{1} q_{2}-\frac{4}{9} \sqrt{3} q_{1}{ }^{2}
\end{aligned}
$$

We use the Theory of Morales-Ruiz- Ramis (see [7] for details) reducing the system to a Normal Variations Equations (NVE) near a non-trivial partial solution. We find a partial solution for $p=p_{1}=p_{2}, q=q_{1}=q_{2}$, and we have

$$
\dot{q}^{2}=-\frac{8}{9} \sqrt{3} q^{3}-4 q^{2}+h,
$$

with solution $\phi(t)=-\frac{\sqrt{3}}{2}-\frac{3 \sqrt{3}}{2} \wp\left(t, g_{2}, g_{3}\right)$, here $\wp$ is Weierstrass p-function with $g_{2}=\frac{4}{3}$, 
$g_{3}=-\frac{4(h-2)}{27}$. Let we put $\eta_{1}=d p_{1}, \eta_{2}=d p_{2}, \xi_{1}=d q_{1}$ and $\xi_{2}=d q_{2}$ then

$$
\begin{aligned}
& \dot{\xi_{1}}=2 \eta_{1}-\eta_{2} \\
& \dot{\xi_{2}}=-\eta_{1}+2 \eta_{2} \\
& \dot{\eta_{1}}=-\left(\frac{8}{3}+\frac{8}{9} \sqrt{3} \cdot \phi\right) \xi_{1}-\left(\frac{4}{3}+\frac{16}{9} \sqrt{3} \cdot \phi\right) \xi_{2} \\
& \dot{\eta_{2}}=-\left(\frac{4}{3}+\frac{16}{9} \sqrt{3} \cdot \phi\right) \xi_{1}-\left(\frac{8}{3}+\frac{8}{9} \sqrt{3} \cdot \phi\right) \xi_{2} .
\end{aligned}
$$

If we get $\xi=\xi_{1}-\xi_{2}$, then we find an equation for NVE $\ddot{\xi}=\left(-\frac{8}{3}+4 \wp\left(t, g_{2}, g_{3}\right)\right) \xi$. This is a Lame-equation with $A=4, B=-\frac{8}{3}$ and in this case we have non Lame-Hermite solutions $A=n(n+1) \neq 4$ for $n \in \mathbf{Z}$. We have non Briochi- Halphen- Crowford solutions $n+1 / 2 \notin \mathbf{N}$ and we have non the Baltassarri solutions $n+1 / 2 \notin \frac{1}{3} \mathbf{Z} \cap \frac{1}{4} \mathbf{Z} \cap \frac{1}{5} \mathbf{Z}-\mathbf{Z}$ - the identity component of its Galois group is non-commutative (see [6] for details).

The theory says that if the identity component of differential Galois group is non-commutative, then the system is not meromorphic integrable (see [7]). This proves that in the case $H_{2}+H_{3}$ there is non additional meromorphic (holomorphic) first integral.

Let us consider the case $\mathrm{H}_{2}+\mathrm{H}_{3}+\mathrm{H}_{4}$ : the Hamiltonian is

$$
\begin{aligned}
\Lambda & ={\tilde{p_{1}}}^{2}-\tilde{p_{1}} \tilde{p_{2}}+{\tilde{p_{2}}}^{2}+\frac{4}{3}{\tilde{q_{1}}}^{2}+\frac{4}{3} \tilde{q_{1}} \tilde{q_{2}}+\frac{4}{3}{\tilde{q_{2}}}^{2}+\frac{4}{9} \sqrt{3}{\tilde{q_{1}}}^{2}{\tilde{q_{2}}} \\
& +\frac{4}{9} \sqrt{3} \tilde{q_{1}}{\tilde{q_{2}}}^{2}+\frac{4}{9}{\tilde{q_{1}}}^{4}+\frac{4}{9}{\tilde{q_{2}}}^{4}+\frac{8}{9}{\tilde{q_{1}}}^{3} \tilde{q_{2}}+\frac{8}{9} \tilde{q_{1}}{\tilde{q_{2}}}^{3}+\frac{4}{3}{\tilde{q_{1}}}^{2}{\tilde{q_{2}}}^{2} .
\end{aligned}
$$

We ignore the tildes and we get the system

$$
\begin{aligned}
\dot{q_{1}} & =2 p_{1}-p_{2} \\
\dot{q_{2}} & =-p_{1}+2 p_{2} \\
\dot{p_{1}} & =-\frac{8}{3} q_{1}-\frac{4}{3} q_{2}-\frac{8}{9} \sqrt{3} q_{1} q_{2}-\frac{4}{9} \sqrt{3} q_{2}{ }^{2} \\
& -\frac{16}{9} q_{1}{ }^{3}-\frac{8}{3} q_{1}{ }^{2} q_{2}-\frac{8}{3} q_{1} q_{2}{ }^{2}-\frac{8}{9} q_{2}{ }^{3} \\
\dot{p_{2}} & =-\frac{4}{3} q_{1}-\frac{8}{3} q_{2}-\frac{8}{9} \sqrt{3} q_{1} q_{2}-\frac{4}{9} \sqrt{3}{q_{1}}^{2} \\
& -\frac{16}{9} q_{2}{ }^{3}-\frac{8}{3} q_{1}{ }^{2} q_{2}-\frac{8}{3} q_{1} q_{2}{ }^{2}-\frac{8}{9} q_{1}{ }^{3} .
\end{aligned}
$$

For a partial solution we get $p=p_{1}=p_{2}, q=q_{1}=q_{2}$ and we find $\psi(t)=-\frac{3 \sqrt{3}}{\sqrt{26} \sinh (2 i t)+1}$.

We get $\eta_{1}=d p_{1}, \eta_{2}=d p_{2}, \xi_{1}=d q_{1}, \xi_{2}=d q_{2}, \xi=\xi_{1}+\xi_{2}$ and we obtain for NVE

$$
\ddot{\xi}=-\left(4+\frac{8}{9} \sqrt{3} \cdot \psi(t)+24 \cdot \psi(t)^{2}\right) \xi .
$$


We need to algebrize (0.11) with a standard change of variable $w=\sqrt{26} \sinh (2 i t)+1$. The result is

$$
\xi^{\prime \prime}=r(w) \xi .
$$

Next we use the Kovacic algorithm to show that (0.12) has non Liouvillian solutions and the identity component of the Galois group of this equation is $S L(2, \mathbf{C})$. This means that the Hamiltonian system (0.10) is non integrable with meromorphic first integrals. This proves that in the case $\mathrm{H}_{2}+\mathrm{H}_{3}+\mathrm{H}_{4}$ there is non additional meromorphic (holomorphic) first integral. The system $H_{3}$ is integrable, $H_{2}+H_{3}$ and $H_{2}+H_{3}+H_{4}$ are non integrable thats why we could conclude that the system $H_{2}+H_{3}+\cdots+H_{k}$ is non integrable for each $k \geq 3$. This proves B).

Acknowledgements. I acknowledge for the partial support by Sofia University Grant 80-10-215/2017.

\section{References}

[1] Dyson F.J., (1962), Statistical theory of the enrgy levels of complex systems, I, II, III, J. Math Phys., 3, 140-156, 157-165, 166-175.

[2] Calogero F., Perelomov A. M., (1978), Properties of Certain Matrices Related to the Equilibrium Configuration of the One-Dimensional Many-Body Problems with the Pair Potentials, $V_{1}(x)=-\log |\sin x|$ and $V_{2}(x)=1 / \sin ^{2} x$, Commun. math. Phys. 59, no.12, 109-116.

[3] Kozlov V.V., (1995), Symmetries, Topology, and Resonace in Hamiltonian Mechanics, Izhevsk, Udmurt Univ. Moskow (in Russian).

[4] Borisov A. V., Kozlov V.V., (1998), Nonitegrability Of a System of Interacting Particles with the Dyson Potential, Doklady Akademii Nauk, Vol 306, no. 1, 30-31.

[5] Duistermaat J. J., (1984), Non-integrability of the 1:1:2- resonance, Ergod. Th. \& Dynam. Sys., 4, 553-568.

[6] Christov O., Georgiev G. (2015), On the integrability of a system describing the stationary solutions in Bose-Fermi mixtures, Chaos Solitons and Fractals, 77, 8, 138-148.

[7] Morales-Ruiz J., (1999), Differential Galois Theory and Non-integrability of Hamiltonian Systems, Birkhäuser .

Georgi Georgiev ggeorgiev3@fmi.uni-sofia.bg

Faculty of Mathematics and Informatics, Sofia University, 1164 Sofia, Bulgaria 